\title{
A Review of Trends in STEM Education Research
}

\author{
Aixa Ḥafṣa, Anggi Cecilia Safaningrum, Fariha Fadhilaturrahmi, \\ Venice Tan Hoay Min, \& Cathryn Li Yuan Ling
}

\begin{abstract}
With the rapid increase in the number of scholarly publications on STEM education in recent years, reviews of the status and trends in STEM education research internationally support the development of the field. For this review, we conducted a systematic analysis of 798 articles in STEM education published between 2000 and the end of 2018 in 36 journals to get an overview about developments in STEM education scholarship. We examined those selected journal publications both quantitatively and qualitatively, including the number of articles published, journals in which the articles were published, authorship nationality, and research topic and methods over the years. The results show that research in STEM education is increasing in importance internationally and that the identity of STEM education journals is becoming clearer over time.
\end{abstract}

Keywords: Journal publication, Literature review, Status, STEM education research, Trends

\section{[A] Introduction}

Conducting systematic reviews to explore the status and trends in specific disciplines is common in educational research. For example, researchers surveyed the historical development of research in mathematics education (Kilpatrick, 1992) and studied patterns in technology usage in mathematics education (Bray \& Tangney, 2017; Sokolowski, Li, \& Willson, 2015). In science education, Tsai and his colleagues have conducted a sequence of reviews of journal articles to synthesize research trends in every 5 years since 1998 (i.e., 1998-2002, 2003-2007, 2008-2012, and 2013-2017), based on publications in three main science education journals including, Science Education, the International Journal of Science Education, and the Journal of Research in Science Teaching (e.g., Lin, Lin, Potvin, \& Tsai, 2019; Tsai \& Wen, 2005). Erduran, Ozdem, and Park (2015) reviewed argumentation in science education research from 1998 to 2014 and Minner, Levy, and Century (2010) reviewed inquiry-based science instruction between 1984 and 2002. There are also many literature reviews and syntheses in engineering and technology education (e.g., Borrego, Foster, \& Froyd, 2015; Xu, Williams, Gu, \& Zhang, 2019). All of these reviews have been well received in different fields of traditional disciplinary education as they critically appraise and summarize the state-of-art of relevant research in a field in general or with a specific focus. Both types of reviews have been conducted with different methods for identifying, collecting, and analyzing relevant publications, and they differ in terms of review aim and topic scope, time period, and ways of literature selection. 
With global recognition of the growing importance of STEM education, we have witnessed the urgent need to support research and scholarship in STEM education (Li, 2014, 2018a). Researchers and educators have responded to this on-going call and published their scholarly work through many different publication outlets including journals, books, and conference proceedings. A simple Google search with the term "STEM," "STEM education," or "STEM education research" all returned more than 450,000,000 items. Such voluminous information shows the rapidly evolving and vibrant field of STEM education and sheds light on the volume of STEM education research. In any field, it is important to know and understand the status and trends in scholarship for the field to develop and be appropriately supported. This applies to STEM education. In this review, we systematically analyze journal publications in STEM education research to overview STEM education scholarship development broadly and globally.

\section{[B] The complexity and ambiguity of examining the status and trends in STEM education research}

A review of research development in a field is relatively straight forward, when the field is mature and its scope can be well defined. Unlike discipline-based education research (DBER, National Research Council, 2012), STEM education is not a well-defined field. Conducting a comprehensive literature review of STEM education research require careful thought and clearly specified scope to tackle the complexity naturally associated with STEM education. In the following sub-sections, we provide some further discussion.

\section{[1] Diverse perspectives about STEM and STEM education}

STEM education as explicated by the term does not have a long history. The interest in helping students learn across STEM fields can be traced back to the 1990s when the US National Science Foundation (NSF) formally included engineering and technology with science and mathematics in undergraduate and K-12 school education (e.g., National Science Foundation, 1998). It coined the acronym SMET (science, mathematics, engineering, and technology) that was subsequently used by other agencies including the US Congress (e.g., United States Congress House Committee on Science, 1998). NSF also coined the acronym STEM to replace SMET (e.g., Christenson, 2011; Chute, 2009) and it has become the acronym of choice. However, a consensus has not been reached on the disciplines included within STEM.

To clarify its intent, NSF published a list of approved fields it considered under the umbrella of STEM (see http://bit.ly/2Bk1Yp5). The list not only includes disciplines widely considered under the STEM tent (called "core" disciplines, such as physics, chemistry, and materials research), but also includes disciplines in psychology and social sciences (e.g., political science, economics). However, NSF's list of STEM fields is inconsistent with other federal agencies. Gonzalez and Kuenzi (2012) noted that at least two US agencies, the Department of Homeland Security and Immigration and Customs Enforcement, use a narrower definition that excludes social sciences. Researchers also view integration across different disciplines of STEM differently using various terms such as, multidisciplinary, interdisciplinary, and transdisciplinary (Vasquez, Sneider, \& Comer, 2013). These are only two examples of the ambiguity and complexity in describing and specifying what constitutes STEM.

Multiple perspectives about the meaning of STEM education adds further complexity to determining the extent to which scholarly activity can be categorized as STEM education. For 
example, STEM education can be viewed with a broad and inclusive perspective to include education in the individual disciplines of STEM, i.e., science education, technology education, engineering education, and mathematics education, as well as interdisciplinary or cross-disciplinary combinations of the individual STEM disciplines (English, 2016; Li, 2014). On the other hand, STEM education can be viewed by others as referring only to interdisciplinary or cross-disciplinary combinations of the individual STEM disciplines (Honey, Pearson, \& Schweingruber, 2014; Johnson, Peters-Burton, \& Moore, 2015; Kelley \& Knowles, 2016; Li, 2018a). These multiple perspectives allow scholars to publish articles in a vast array and diverse journals, as long as journals are willing to take the position as connected with STEM education. At the same time, however, the situation presents considerable challenges for researchers intending to locate, identify, and classify publications as STEM education research. To tackle such challenges, we tried to find out what we can learn from prior reviews related to STEM education.

\section{[2] Guidance from prior reviews related to STEM education}

A search for reviews of STEM education research found multiple reviews that could suggest approaches for identifying publications (e.g., Brown, 2012; Henderson, Beach, \& Finkelstein, 2011; Kim, Sinatra, \& Seyranian, 2018; Margot \& Kettler, 2019; Minichiello, Hood, \& Harkness, 2018; Mizell \& Brown, 2016; Thibaut et al., 2018; Wu \& Rau, 2019). The review conducted by Brown (2012) examined the research base of STEM education. He addressed the complexity and ambiguity by confining the review with publications in eight journals, two in each individual discipline, one academic research journal (e.g., the Journal of Research in Science Teaching) and one practitioner journal (e.g., Science Teacher). Journals were selected based on suggestions from some faculty members and K-12 teachers. Out of 1100 articles published in these eight journals from January 1, 2007, to October 1, 2010, Brown located 60 articles that authors self-identified as connected to STEM education. He found that the vast majority of these 60 articles focused on issues beyond an individual discipline and there was a research base forming for STEM education. In a follow-up study, Mizell and Brown (2016) reviewed articles published from January 2013 to October 2015 in the same eight journals plus two additional journals. Mizell and Brown used the same criteria to identify and include articles that authors self-identified as connected to STEM education, i.e., if the authors included STEM in the title or author-supplied keywords. In comparison to Brown's findings, they found that many more STEM articles were published in a shorter time period and by scholars from many more different academic institutions. Taking together, both Brown (2012) and Mizell and Brown (2016) tended to suggest that STEM education mainly consists of interdisciplinary or cross-disciplinary combinations of the individual STEM disciplines, but their approach consisted of selecting a limited number of individual discipline-based journals and then selecting articles that authors self-identified as connected to STEM education.

In contrast to reviews on STEM education, in general, other reviews focused on specific issues in STEM education (e.g., Henderson et al., 2011; Kim et al., 2018; Margot \& Kettler, 2019; Minichiello et al., 2018; Schreffler, Vasquez III, Chini, \& James, 2019; Thibaut et al., 2018; Wu \& Rau, 2019). For example, the review by Henderson et al. (2011) focused on instructional change in undergraduate STEM courses based on 191 conceptual and empirical journal articles published between 1995 and 2008. Margot and Kettler (2019) focused on what is known about teachers' values, beliefs, perceived barriers, and needed support related to STEM education based on 25 empirical journal articles published between 2000 and 2016. The focus of these reviews allowed the researchers to limit the number of articles considered, 
and they typically used keyword searches of selected databases to identify articles on STEM education. Some researchers used this approach to identify publications from journals only (e.g., Henderson et al., 2011; Margot \& Kettler, 2019; Schreffler et al., 2019), and others selected and reviewed publications beyond journals (e.g., Minichiello et al., 2018; Thibaut et al., 2018; Wu \& Rau, 2019).

\section{[3] Summary}

The discussion in this section suggests possible reasons contributing to the absence of a general literature review of STEM education research and development: (1) diverse perspectives in existence about STEM and STEM education that contribute to the difficulty of specifying a scope of literature review, (2) its short but rapid development history in comparison to other discipline-based education (e.g., science education), and (3) difficulties in deciding how to establish the scope of the literature review. With respect to the third reason, prior reviews have used one of two approaches to identify and select articles: (a) identifying specific journals first and then searching and selecting specific articles from these journals (e.g., Brown, 2012; Erduran et al., 2015; Mizell \& Brown, 2016) and (b) conducting selected database searches with keywords based on a specific focus (e.g., Margot \& Kettler, 2019; Thibaut et al., 2018). However, neither the first approach of selecting a limited number of individual discipline-based journals nor the second approach of selecting a specific focus for the review leads to an approach that provides a general overview of STEM education scholarship development based on existing journal publications.

\section{[4] Current review}

Two issues were identified in setting the scope for this review.

1) What time period should be considered?

2) What publications will be selected for review?

\section{[5] Time period}

We start with the easy one first. As discussed above, the acronym STEM did exist until the early 2000s. Although the existence of the acronym does not generate scholarship on student learning in STEM disciplines, it is symbolic and helps focus attention to efforts in STEM education. Since we want to examine the status and trends in STEM education, it is reasonable to start with the year 2000. Then, we can use the acronym of STEM as an identifier in locating specific research articles in a way as done by others (e.g., Brown, 2012; Mizell \& Brown, 2016). We chose the end of 2018 as the end of the time period for our review that began during 2019.

\section{[6] Focusing on publications beyond individual discipline-based journals}

As mentioned before, scholars responded to the call for scholarship development in STEM education with publications that appeared in various outlets and diverse languages, including journals, books, and conference proceedings. However, journal publications are typically credited and valued as one of the most important outlets for research exchange (e.g., Erduran et al., 2015; Henderson et al., 2011; Lin et al., 2019; Xu et al., 2019). Thus, in this review, we will also focus on articles published in journals in English. 
The discourse above on the complexity and ambiguity regarding STEM education suggests that scholars may publish their research in a wide range of journals beyond individual discipline-based journals. To search and select articles from a wide range of journals, we thought about the approach of searching selected databases with keywords as other scholars used in reviewing STEM education with a specific focus. However, existing journals in STEM education do not have a long history. In fact, IJ-STEM is the first journal in STEM education that has just been accepted into the Social Sciences Citation Index (SSCI) (Li, 2019a). Publications in many STEM education journals are practically not available in several important and popular databases, such as the Web of Science and Scopus. Moreover, some journals in STEM education were not normalized due to a journal's name change or irregular publication schedule. For example, the Journal of STEM Education was named as Journal of SMET Education when it started in 2000 in a print format, and the journal's name was not changed until 2003, Vol 4 (3 and 4), and also went fully on-line starting 2004 (Raju \& Sankar, 2003). A simple Google Scholar search with keywords will not be able to provide accurate information, unless you visit the journal's website to check all publications over the years. Those added complexities prevented us from taking the database search as a viable approach. Thus, we decided to identify journals first and then search and select articles from these journals.

\section{[7] Research questions}

Given a broader range of journals and a longer period of time to be covered in this review, we can examine some of the same questions as the IJ-STEM review (Li, Froyd, \& Wang, 2019), but we do not have access to data on readership, articles accessed, or articles cited for the other journals selected for this review. Specifically, we are interested in addressing the following six research questions:

1) What were the status and trends in STEM education research from 2000 to the end of 2018 based on journal publications?

2) What were the patterns of publications in STEM education research across different journals?

3) Which countries or regions, based on the countries or regions in which authors were located, contributed to journal publications in STEM education?

4) What were the patterns of single-author and multiple-author publications in STEM education?

5) What main topics had emerged in STEM education research based on the journal publications?

6) What research methods did authors tend to use in conducting STEM education research?

\section{[C]Method}

Based on the above discussion, we developed the methods for this literature review to follow careful sequential steps to identify journals first and then identify and select STEM education research articles published in these journals from January 2000 to the end of 2018. The methods should allow us to obtain a comprehensive overview about the status and trends of STEM education research based on a systematic analysis of related publications from a broad range of journals and over a longer period of time. 